\title{
Beyond spatial correlation effect in micro-Raman light scattering: An example of zinc-blende GaN/GaAs hetero-interface
}

\author{
J. Q. Ning, ${ }^{1, a)}$ C. C. Zheng, ${ }^{1,2}$ L. X. Zheng, ${ }^{3}$ and S. J. Xu ${ }^{1, b)}$ \\ ${ }^{1}$ Department of Physics, HKU-Shenzhen Institute of Research and Innovation (HKU-SIRI), \\ The University of Hong Kong, Pokfulam Road, Hong Kong, China \\ ${ }^{2}$ Mathematics and Physics Centre, Department of Mathematical Sciences, Xi' an Jiaotong-Liverpool \\ University, Suzhou 215123, China \\ ${ }^{3}$ Department of Mechanical Engineering, Khalifa University, P.O. Box: 127788, Abu Dhabi, \\ United Arab Emirates
}

(Received 19 March 2015; accepted 3 August 2015; published online 17 August 2015)

\begin{abstract}
Spatially resolved Raman light scattering experiments were performed on a zinc-blende GaN/GaAs heterostructure with confocal micro-Raman scattering technique under the backscattering geometric configuration. By varying the illumination spot locations across the heterostructure interface, we found that the Raman light scattering spectral features change remarkably. The interface effect on the GaAs substrate manifested as a much broader lineshape of the transverse optical (TO) phonon mode. Two kinds of broadening mechanisms, namely, spatial correlation induced wave-vector relaxation effect and lattice-mismatch strain + compositional intermixing effect, have been identified. The former leads to the broadening of the TO mode at the low-energy side, whereas the latter accounts for the broadening at the high-energy side. The diffuse light scattering from the highly defective nucleation layer of $\mathrm{GaN}$ was found to produce a broad scattering background of the GaN TO mode. The methodology and conclusions of the present work could be applicable to Raman spectroscopic studies on other material interfaces. (C) 2015 AIP Publishing LLC.

[http://dx.doi.org/10.1063/1.4928618]
\end{abstract}

\section{INTRODUCTION}

Raman light scattering is one kind of fundamental light-matter interaction phenomenon. For defective or even microcrystalline semiconductors, their Raman light scattering peaks exhibit asymmetric broadening at the lower energy side and redshift in spectral peak. In order to interpret these spectral changes, a so-called "spatial correlation" model was proposed. ${ }^{1-3}$ In this model, a nanometer scale length parameter, namely, correlation length, was adopted to characterize "spatial correlation" in defective semiconductors. As disorder degree, i.e., defect density, increases in semiconductors, this characteristic length reduces like the mean free path of electron transport. From available application practice, the "spatial correlation" model has been successfully used to explain the observed asymmetric broadening at the lower energy side of the spectral lineshape and the redshift in spectral peak for Raman light scattering from defective and microcrystalline semiconductors.

Moreover, the study of semiconductor hetero-interfaces has long been an important issue in both materials science and device physics. From the point of view of device physics, such hetero-interface may be a device, ${ }^{4}$ and the design of new interface parameters and the achievement of novel interfaces may greatly improve the performance of existing devices while also making new kinds of devices

\footnotetext{
a) Present address: Suzhou Institute of Nano-tech and Nano-bionics, Chinese Academy of Sciences, Suzhou 215123, China.

${ }^{b)}$ Author to whom correspondence should be addressed. Electronic mail: sjxu@hku.hk
}

possible. In the field of materials research, the control of interface growth is a key step to the acquisition of high crystallographic quality and desired material properties. Group III-V compound semiconductors have wide applications in optoelectronic devices such as laser diodes (LDs) $)^{5}$ and lightemitting diodes (LEDs), ${ }^{6,7}$ with heterostructures generally included in most compound semiconductor devices. For example, $\mathrm{GaN}$ and relevant III-nitrides have been developed for fabricating blue and short-wavelength LEDs and LDs. ${ }^{5}$ Currently, they are usually grown on foreign substrates such as sapphire, $\mathrm{SiC}, \mathrm{GaAs}$, and $\mathrm{Si}$ due to the lack of large size $\mathrm{GaN}$ bulk crystal. GaN is generally crystallized in a wurtzite crystal structure because the hexagonal phase is thermodynamically stable. Compared with the wurtzite structure, cubic GaN with a zinc-blende structure is metastable. Although it does not exist in nature, it can be artificially grown under appropriate conditions. ${ }^{7}$

The investigation of the cubic phase of compound semiconductors is of broad interest due to their wide-ranging applications in different fields and some application advantages over the hexagonal phase, such as larger optical gain and lower threshold current density. ${ }^{8}$ In the case of GaN, its cubic phase is predicted to have more attractive features such as higher carrier mobility ${ }^{9}$ and easier p-type doping. ${ }^{10}$ The growth of cubic GaN can be achieved by using a cubic GaAs substrate and nonequilibrium epitaxial techniques. $^{7,11,12}$ The knowledge of the hetero-interface between the grown cubic GaN epilayer and GaAs substrate is of both scientific and technological interest. In particular, the structural information about the GaN/GaAs interface from 
the microscopic level may lead to a recognition of the local atomic arrangements which is expected to provide valuable insight into the growth mechanisms and interface-related material properties. The structural analysis of the cubic GaN/ GaAs interfaces has been performed by means of various $\mathrm{X}$-ray and electron diffraction techniques so far $^{7,11,12}$ which deliver useful crystallographic details. Additionally, being a widely recognized powerful optical method, Raman scattering, especially confocal micro-Raman scattering, could provide richer and more in-depth information about GaN heteroepilayers. ${ }^{13,14}$ However, to the best of our knowledge, there have been very few spatially resolved Raman studies of the compound semiconductor heterostructure interfaces. In this article, we apply optical micro-Raman scattering to the investigation of a cubic GaN/GaAs interface obtained from a high quality cubic GaN epilayer deposited on a GaAs (001) substrate using metalorganic vapor phase epitaxy (MOVPE). A remarkable interface effect on the Raman modes of both GaAs substrate and GaN epilayer is revealed. Spectral lineshapes of the characteristic Raman modes, especially the TO mode of GaAs, are unveiled to be intimately correlated to the microscopic structures of the heterointerface.

\section{MATERIALS AND METHODS}

The cubic GaN/GaAs heterostructures investigated in the present study were prepared by growing GaN epilayers on GaAs (001) substrates with MOVPE. During the growth of the GaN epilayers, trimethylgallium (TMGa) and ammonia $\left(\mathrm{NH}_{3}\right)$ were used as the $\mathrm{Ga}$ and $\mathrm{N}$ precursors, respectively, with hydrogen as the carrier gas. Typical growth sequence of the samples is described as follows. A GaN buffer layer with thickness of $20 \mathrm{~nm}$ was first deposited at $500^{\circ} \mathrm{C}$ with a $2 \mu \mathrm{m}$ $\mathrm{GaN}$ epilayer grown at $820^{\circ} \mathrm{C}$. Raman scattering measurements were carried out at room temperature on a confocal microscope system using backscattering geometry with polarization analysis. The $488.0 \mathrm{~nm}$ line of a Coherent $\mathrm{Ar}^{+}+\mathrm{Kr}^{+}$ mixed gas laser was employed as the excitation light, while a $100 \times$ objective lens was used to focus the excitation laser beam as well as collect the scattered Raman light signal which was dispersed by a grating of 1800 lines $/ \mathrm{mm}$ in a SPEX 750 monochromator and finally detected by a Princeton SpectrumOne charge coupled device detector cooled by liquid nitrogen. Under these conditions, the spatial resolution of the instrument is about $1 \mu \mathrm{m}$. In the microRaman spectroscopic measurements, the excitation power was kept at $\sim 10 \mathrm{~mW}$. The X-ray diffraction (XRD) measurements of the samples were performed on a Rigaku SLX-1AL double-crystal diffractometer employing $\mathrm{Cu} K \alpha 1$ radiation from a rotating-anode source + a Ge (004) monochromator as the excitation source. ${ }^{7}$

\section{RESULTS AND DISCUSSION}

Figure 1 shows the XRD patterns recorded from an as-grown GaN epilayer on GaAs (001) substrate. Both the diffraction patterns from the GaN epilayer and GaAs substrate can be easily identified. The (002) diffraction of cubic GaAs appears as a narrow peak, while that of cubic $\mathrm{GaN}$ is

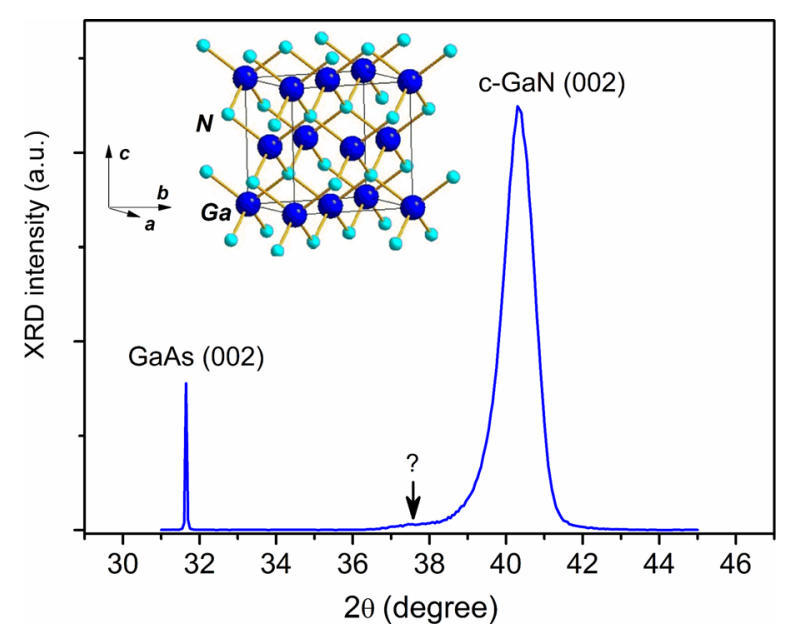

FIG. 1. X-ray diffraction patterns of the cubic GaN/GaAs (001) sample. The cubic lattice structure majority of the GaN epilayer is clearly indicated by the (002) diffraction pattern centered at $40.3^{\circ}$. In addition, a shoulder structure (marked by a vertical arrow) is observed at the lower-angle side of the cubic GaN (002) diffraction pattern. The inset depicts a schematic lattice structure of cubic GaN with zinc-blende structure.

relatively broad. The (002) diffraction pattern of cubic $\mathrm{GaN}$ was centered at $40.3^{\circ}$ which is in line with previously reported values. ${ }^{7,11}$ The nature of the high-quality cubic GaN phase has also been confirmed by the photoluminescence studies reported in the literature. ${ }^{15}$ The broad diffraction pattern from the GaN epilayer is actually the reflection of fluctuations in lattice constant in the epilayer, indicating the variation of atomic long distance ordering in the epilayer from the bottom interface to the top surface. In addition, we also observe a weak shoulder structure, marked with a vertical arrow in Fig. 1, at the low-angle side of the GaN (002) main peak. This shoulder corresponds to a larger lattice constant and will be discussed later in the analysis of the Raman scattering results.

Figures 2 and 3 depict the Raman spectra measured at different sites of the sample and with different polarization configurations. We employ a four-letter notation for the propagation and polarization setups. The four-letter symbol,

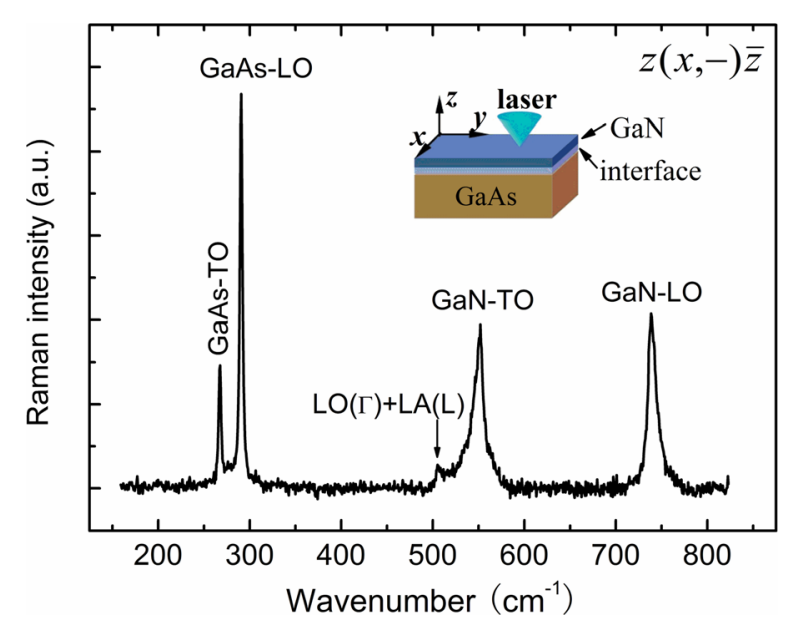

FIG. 2. Raman spectrum recorded from the heterostructure under the $z(x,-) \bar{z}$ geometric configuration. The inset shows a schematic illustration of the geometric configuration for the measurement of this spectrum. 


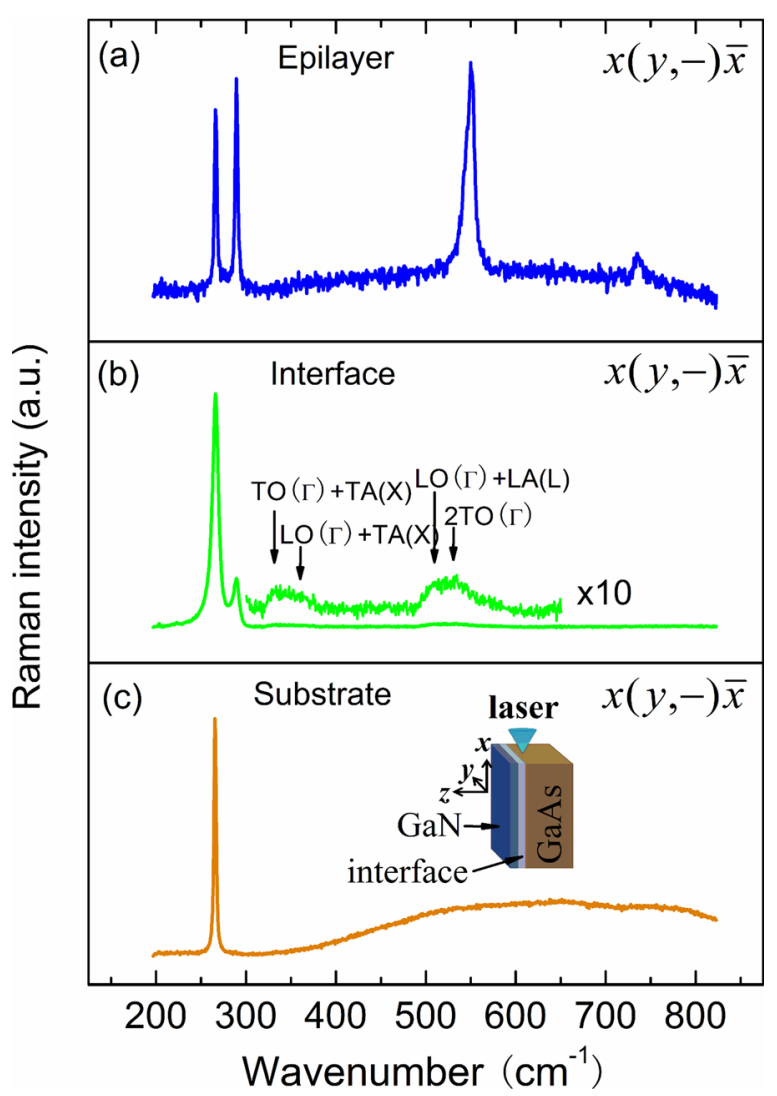

FIG. 3. Raman spectra registered under the $x(y,-) \bar{x}$ configuration while the excitation laser the illumination point is located on (a) the side of GaN epilayer, (b) the interface region, and (c) the side of GaAs substrate. The inset depicts a schematic diagram of the geometric configuration.

$z(x,-) \bar{z}$, represents a geometric configuration in which $z(-,-) \bar{z}$ means incident light along the $z$ direction and the scattered light along the $-z$ direction, and $-(x,-)-$ means the polarization plane of incident light parallel to the $x$ axis and the polarization of the scattered light not analyzed. Likewise, $x(y,-) \bar{x}$ denotes a backscattering configuration along the $x$ axis, the incident light polarized along the $y$ axis, and no polarization analysis for the scattered light. In the measurements, we define the $z$ axis as parallel to [001] direction of the sample, and $x$ and $y$ along the [100] and [010], respectively. From the Raman spectrum in Fig. 2, which is recorded in $z(x,-) \bar{z}$ geometry, we can see that all the optical phonon modes from both the GaAs substrate and the GaN epilayer are excited and recorded. The GaAs substrate's Raman spectrum exhibits a strong longitudinal optical (LO) mode at $291 \mathrm{~cm}^{-1}$ and a relatively weaker TO mode at $267 \mathrm{~cm}^{-1}$. Additionally, a second-order weak Raman scattering band at $506 \mathrm{~cm}^{-1}$ is also observed for $\mathrm{LO}(\Gamma)+\mathrm{LA}(\mathrm{L}) .{ }^{16}$ For the GaN epilayer, its TO mode is centered at $552 \mathrm{~cm}^{-1}$ with its LO mode at $739 \mathrm{~cm}^{-1}$, consistent with the reported values for the cubic phase of GaN. ${ }^{15,17}$ Unlike the situation of the TO and LO modes of GaAs substrate, the intensities of TO and LO modes of GaN epilayer are comparable. Moreover, their line widths are much larger than those of the GaAs substrate. The broadened spectral feature is related to the lattice fluctuation effect in the GaN epilayer, whereas the comparable TO and LO Raman intensities are due to the interface effect which will be analyzed later.
To identify the interface effects, measurements of the backscattering configuration along the $x$ axis were performed. By carefully scanning the laser spot in the $\mathrm{z}$ direction across the GaN/GaAs interface, the location of the point of illumination at the interface can be easily determined by monitoring the emergence of the forbidden LO phonon mode of the GaAs substrate. Figure 3 shows the spatially resolved Raman spectra recorded in $x(y,-) \bar{x}$ scattering geometry upon selective points of illumination, where (a) is the spectrum for the point of illumination on the side of the GaN epilayer, (b) on the interface region, and (c) on the side of the GaAs substrate. We first inspect the Raman modes of the GaAs substrate. According to the polarization selection rule for the Ramanactive modes in a zinc-blende structure, the TO mode is allowed, but the LO mode is forbidden in the $x(y,-) \bar{x}$ scattering geometry, ${ }^{18}$ which is proven by the Raman scattering result in Fig. 3(c). However, as we can see in Figs. 3(a) and 3(b), the LO mode gets observable, while its intensity becomes even greater than that of the TO mode as shown in Fig. 3(a). Since all three spectra were recorded in the $x(y,-) \bar{x}$ geometric configuration, the observation of the LO mode is a fascinating result, which deserves a brief discussion. Theoretically, as mentioned earlier, Raman scattering by the LO mode should be totally forbidden in the $x(y,-) \bar{x}$ backscattering geometry. This is well confirmed by the Raman spectrum in Fig. 3(c) when the laser beam is focused on the GaAs substrate side. When the laser illuminating point is moved from the GaAs substrate to the interface region, the situation changes significantly because the laser beam may experience a strong diffuse scattering/reflection from the highly disordered interface layer where strong atomic disorder, intermixing, and a dislocation network exist. A portion of the scattered laser beam may propagate into the GaAs substrate along the non- $x$ direction, which may excite phonons with wave vector apart from the $x$ direction, i.e., LO mode phonons. Meanwhile, the polarization of the scattered laser beam may also be altered. As a result, the GaAs LO phonon mode becomes detectable. We therefore propose a tentative name of "interface effect" to this phenomenon. Such an effect may generally exist in Raman scattering experiments to some extent if the incident light is experiencing a significant scattering due to a rough surface or highly defective interfaces. When the laser illuminating point is moved further from the $\mathrm{GaN} / \mathrm{GaAs}$ interface to the GaN epilayer, the Raman intensity of GaAs LO mode becomes even stronger than that of GaAs TO mode. Similarly, as expected, a strong TO mode of the epilayer is observed due to the allowed transition and large scattering cross section, while the originally forbidden LO mode of the epilayer in the $x(y,-) \bar{x}$ backscattering geometry is also detectable although its intensity is weak. This could be explained by the relaxed selection rule to some extent induced by high density of defects/dislocations in the GaN epilayer. Such an explanation is jointly supported by the significantly broadened XRD peak and TO phonon mode. Actually, the appearance of the forbidden LO mode in the Raman spectrum of the $\mathrm{GaN}$ epilayer can still be ascribed to the "interface effect" - one kind of diffuse scattering. More fascinatingly, both the TO and LO modes of GaAs substrate are observed in the Raman spectrum, despite the fact that the laser beam is 
focused on the side of the GaN epilayer. This anomalous phenomenon could be explained in terms of the narrow thickness (intentionally grown to a thickness of $2 \mu \mathrm{m}$ ) of the epilayer and the divergence propagation of the focused laser beam beyond its short focus length. The GaN modes cannot be seen in Figs. 3(b) and 3(c). This is most likely because the $488.0 \mathrm{~nm}$ excitation light is highly transparent for $\mathrm{GaN}$ but could be significantly absorbed by GaAs, leading to fewer photons for diffuse scattering.

Likewise, due to the interface effect, we can explain the strongly enhanced GaN TO mode intensity as observed in Fig. 2. In the $z(x,-) \bar{z}$ geometric configuration, most of the $488.0 \mathrm{~nm}$ incident photons pass through the cubic GaN epilayers arriving at the $\mathrm{GaN} / \mathrm{GaAs}$ interface layer which accommodates large amounts of coarseness or interface disorders and a high density of defects of various kinds. ${ }^{11,12,19}$ At this highly defective interface, diffuse reflection/scattering of the incident photons occurs. After the diffuse reflection, many of the incident photons shall propagate inside the $\mathrm{GaN}$ epilayer in non- $z$ direction, which may experience effective Raman scattering by the GaN TO phonons. Consequently, substantial enhancement is observed for the GaN TO mode in the Raman scattering spectrum. Similarly, an enhancement effect is observed for the TO mode of GaAs substrate due to the scattering of photons with non- $z$ direction momenta. After proposing an interface scattering picture and conducting some qualitative arguments, we will attempt to give a detailed spectral analysis of the Raman spectra in the following paragraph.

From a careful examination of a portion of the Raman spectrum recorded from the $\mathrm{GaN} / \mathrm{GaAs}$ interface shown in Fig. 3(b), we can observe some second-order Raman scattering bands of the GaAs substrate, including $\mathrm{TO}(\Gamma)+\mathrm{TA}(\mathrm{X})$, $\mathrm{LO}(\Gamma)+\mathrm{TA}(\mathrm{X})$, and $2 \mathrm{TO}(\Gamma)$ structures. ${ }^{16}$ This indicates that some $\mathrm{N}$ atoms already incorporate into GaAs to form an interface layer. No Raman mode of the GaN epilayer is detected, whereas asymmetrically broadened TO and LO modes of the GaAs substrate are recorded. It should be noted that when the laser beam is located on the sides of the GaN epilayer and GaAs substrate, respectively, both the TO and LO modes of GaAs are sharp and symmetric which reflects the excellent crystalline nature of the GaAs substrate used for the growth of GaN epilayer. For a better comparison, we redraw the TO spectral lines of GaAs substrate in Figs. 3(b) and 3(c) as the top and bottom curves (solid circles) in Fig. 4(a), respectively. As fitted with a Gaussian lineshape function, the TO mode, recorded when the point of illumination is focused on the side of GaAs substrate, exhibits a symmetrical and narrow lineshape with a full width at half maximum (FWHM) of $2.7 \mathrm{~cm}^{-1}$. As for the TO phonon peak recorded for the point of illumination at the interface, it cannot be fitted by a simple Gaussian lineshape function, as indicated by the green line. It possesses a large FWHM of $7 \mathrm{~cm}^{-1}$ and an asymmetric lineshape with a significant broadening of the low-energy tail.

To interpret the observed broadening of the GaAs TO mode, we first consider the effect of wave-vector relaxation. It is well known that, in an infinite crystal, only the optical phonon modes at the center of the Brillouin zone (wave-vector $q \approx 0$ ) may be observed in Raman spectroscopic

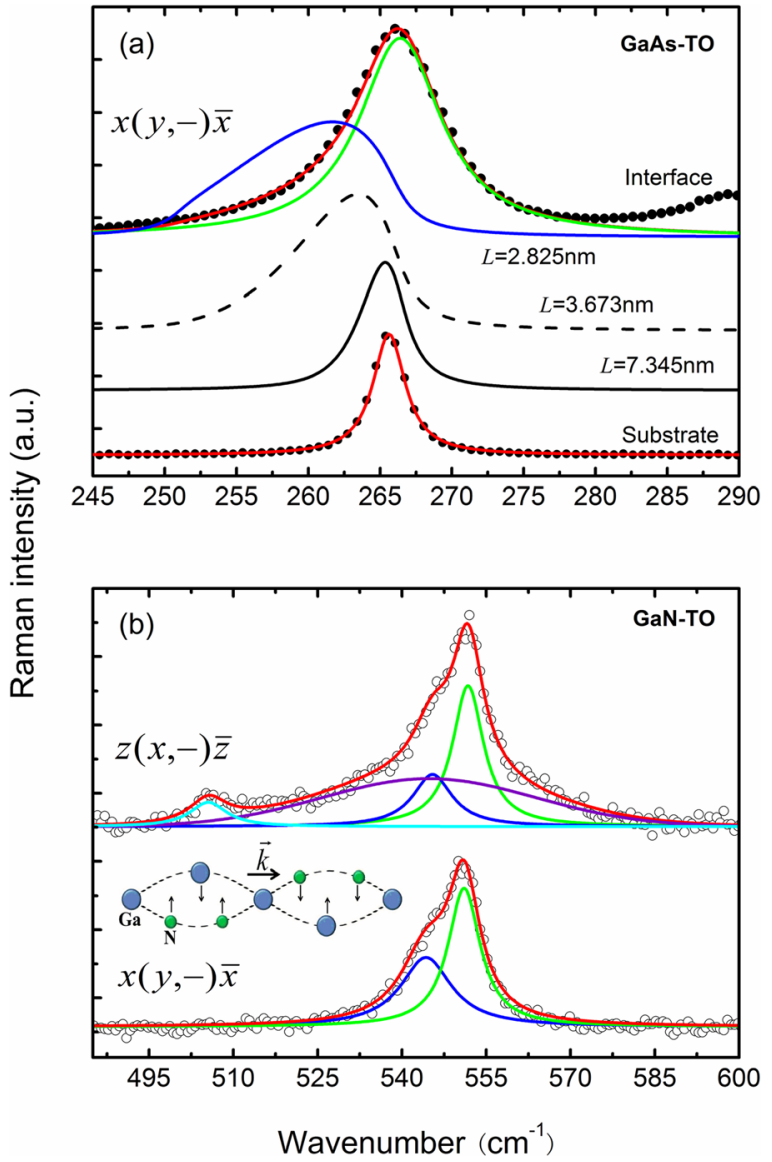

FIG. 4. (a) Comparison of the GaAs TO phonon spectral lines recorded from the substrate (dots at bottom) and the interface (dots at top), as well as the theoretical curves by using the spatial-correlation model and a Gaussian lineshape function. (b) Comparison of the GaN TO phonon spectral lines acquired under the $z(x,-) \bar{z}$ (circles at top) and $x(y,-) \bar{x}$ (circles at bottom) geometric configurations. The experimental Raman spectra were decomposed with Gaussian lineshape function with the best fitting obtained by using the Nonlinear Least Squares Fitting (NLSF) method based on the Levenberg-Marquardt (LM) algorithm.

measurements due to the rule of crystal momentum conservation. When a crystal becomes imperfect, such as through the incorporation of microcrystallite boundaries and defects, the translational symmetry of the crystalline lattice is disrupted. This may result in the localization of phonon wave function to a finite extent otherwise known as the phonon confinement phenomenon. ${ }^{1-3}$ As a result, the relaxation of the momentum conservation rule in Raman scattering of crystal phonons occurs, allowing phonons with $q>0$ to contribute to the Raman scattering lineshape. A direct consequence of the wave-vector relaxation is the softening (redshift) of the phonon energy and asymmetric broadening of the spectral lines. We then employ the spatial-correlation mode ${ }^{17-19}$ to examine how the wave-vector relaxation contributes to the broadening of the TO phonon of GaAs when the laser beam is focused at the interface. With the assumption of a spherical region confining the phonon, the Raman intensity can be written as a function of the frequency $\omega$ (Refs. 2 and 3)

$$
I(\omega) \propto \int_{0}^{1} \exp \left(\frac{-q^{2} L^{2}}{4}\right) \frac{d^{3} q}{[\omega-\omega(q)]^{2}+\left(\Gamma_{0} / 2\right)^{2}},
$$


where $\omega(q)$ is the phonon dispersion curve, $\Gamma_{0}$ is the natural width, and $L$ is the correlation length. Wave-vector $q$ is expressed in units of $2 \pi / a$, where $a$ is the lattice constant (5.65 $\AA$ for GaAs). For the TO dispersion curve $\omega(q)$ of GaAs, an analytical model relation is adopted ${ }^{2}$

$$
\omega(q)=A+B \cos (\pi q),
$$

where $A=258.7 \mathrm{~cm}^{-1}$ and $B=7.6 \mathrm{~cm}^{-1}$ obtained from the actual dispersion of the TO phonon in GaAs. ${ }^{20}$ As shown in Fig. 4(a), the solid black, dashed black, and blue lines are the theoretical curves calculated by using Eqs. (1) and (2), with correlation length $L=7.345,3.673$, and $2.825 \mathrm{~nm}$, respectively.

From the theoretical curves shown in Fig. 4(a), we can see that, as the correlation length decreases (i.e., the disordering degree increases), the TO Raman spectral line shifts toward the lower energy direction while asymmetrically broadening on the lower energy side. In other words, the wave-vector relaxation only results in a redshift in energy and asymmetric broadening on the lower energy side provided that the energy dispersion of TO phonon is described by Eq. (2). By comparing the spectral lines shown in Fig. 4(a), we can conclude that the wave-vector relaxation effect alone is not enough to explain the overall broadening of the GaAs TO Raman line at the interface. Instead, we have to take into account additional physical mechanisms to interpret the observed "interface effect." In fact, an interface between two kinds of semiconductors, including the case of the $\mathrm{c}-\mathrm{GaN} / \mathrm{GaAs}$ heterostructure investigated in the present study, could be much more complicated than the defective crystals to which the spatial correlation model applies. In a hetero-interface between two different kinds of crystals with different lattices and atomic species, large lattice-mismatch strain, ${ }^{21}$ significant compositional intermixing, ${ }^{22}$ and a high degree of atomic disorder ${ }^{1}$ could exist. It is known that cubic $\mathrm{GaN}$ has a much smaller lattice constant $(4.52 \AA)$ than GaAs $(5.65 \AA)$. In other words, the lattice mismatch between c-GaN and GaAs is as large as $20 \%$. The smaller lattice constant of c-GaN will cause a compressive stress in the interfacial lattice of the GaAs substrate, which results in a blueshift of GaAs phonon energy. ${ }^{21}$ Moreover, light $\mathrm{N}$ atoms may enter the interfacial lattice of GaAs substrate by substituting much heavier As atoms during the deposition of c-GaN, leading to a reduction of the averaged atomic mass. This could be another factor causing the blueshift of GaAs phonon frequency. Therefore, we must consider at least two different mechanisms, namely, the wave-vector relaxation which accounts for the broadening at the lower energy side and the lattice-mismatch strain and compositional intermixing that causes the broadening at the higher energy side to interpret the experimental overall broadening of GaAs TO Raman line at the interface of the c-GaN/GaAs heterostructure. For the first mechanism, it is mathematically represented by Eq. (1). For the second mechanism, we adopt a standard Gaussian lineshape function to describe it quantitatively. By doing so, we can obtain an excellent simulation to the overall TO spectral line of the GaAs substrate, as shown by a red solid line in the top group of spectral lines in Fig. 4(a).
Next, we turn to inspect the lineshapes of the GaN TO phonon in Fig. 4(b), corresponding to the peaks in Figs. 2 and 3(a) accordingly. From the Raman spectrum registered under $x(y,-) \bar{x}$ geometric configuration, the TO phonon line exhibits a double-component structure, which can be decomposed into two Gaussian spectral lines centered at $\sim 552$ and $545 \mathrm{~cm}^{-1}$, respectively, by using the Nonlinear Least Squares Fitting (NLSF) method based on the Levenberg-Marquardt (LM) algorithm. The peak at $552 \mathrm{~cm}^{-1}$ corresponds to the standard TO phonon energy of cubic $\mathrm{GaN}$, but that at $545 \mathrm{~cm}^{-1}$ is a novel one that is of neither cubic GaN nor hexagonal GaN. ${ }^{12,23}$ We therefore tentatively attribute it to a strain-related TO mode of the interfacial lattice of c-GaN. In the $\mathrm{c}-\mathrm{GaN}$ interfacial lattice region, a tensile stress may exist due to the smaller lattice constant of c-GaN with respect to that of GaAs substrate. Such a statement is positively supported by the shoulder structure in the low-angle side of the main $\mathrm{GaN}$ (002) diffraction peak in Fig. 1. Tensile stress shall result in a redshift of phonon energy.

Finally, we discuss the difference between the Raman spectral lines of GaN TO mode registered under $z(x,-) \bar{z}$ and $x(y,-) \bar{x}$ geometric configurations. In addition to the secondorder Raman scattering structure at $506 \mathrm{~cm}^{-1}$ of the GaAs substrate, a very broad background (violet solid line) of light scattering can be seen for the $z(x,-) \bar{z}$ configuration. The appearance of the second-order Raman scattering from the GaAs substrate indicates that a large portion of the excitation laser beam of $488.0 \mathrm{~nm}$ can travel across the interface of $\mathrm{GaN} / \mathrm{GaAs}$ and illuminate the substrate. As argued earlier, the interfacial layer of $\mathrm{GaN} / \mathrm{GaAs}$ is highly defective and stained. Yet again, we attribute this broad background to the "interface effect"- a complicated scattering of laser beam by the interface layer between two kinds of semiconductors. Such complicated light scattering may be one kind of diffuse light scattering, for example, diffuse X-ray scattering by disordered crystals. ${ }^{24} \mathrm{We}$ are conducting further analysis of this phenomenon.

\section{CONCLUSION}

In summary, we have demonstrated a practical application of spatially resolved micro-Raman scattering to the study of hetero-interface in a cubic GaN/GaAs system. A comprehensive effect, namely, interface effect taking into account at least wave-vector relaxation and lattice-mismatch strain + compositional intermixing, is proposed to interpret the broadening and frequency shift of GaAs and GaN TO phonon modes registered under different geometric configurations. The spectral analytic methodology and conclusions could be general and applicable to the precise Raman investigations on semiconductor hetero-interfaces.

\section{ACKNOWLEDGMENTS}

This work was financially supported by HK-RGC-GRF Grants (Grant No. HKU 705812P), Shenzhen Municipal Science and Technology Innovation Council (Contract No. JCYJ20120615142933076), and the SRT on New Materials of HKU. Partial funding was also provided by a grant from the University Grants Committee Areas of Excellence 
Scheme of the Hong Kong Special Administrative Region, China (Project No. [AoE/P-03/08]). C. C. Zheng acknowledges the support from XJTLU by a Research Development Fund grant (RDF-12-02-03) and the English language proof reading by $\mathrm{E}$. Touchstone and L. N. Shelmerdine from Language Centre of XJTLU.

${ }^{1}$ H. Richter, Z. P. Wang, and L. Ley, Solid State Commun. 39, 625 (1981).

${ }^{2}$ K. K. Tiong, P. M. Amirtharaj, F. H. Pollak, and D. E. Aspnes, Appl. Phys. Lett. 44, 122 (1984).

${ }^{3}$ P. Parayanthal and F. H. Pollak, Phys. Rev. Lett. 52, 1822 (1984).

${ }^{4}$ H. Kroemer, Rev. Mod. Phys. 73, 783 (2001).

${ }^{5}$ S. Nakamura, G. Fasol, and S. J. Pearton, The Blue Laser Diodes (Springer, Berlin, 2000).

${ }^{6}$ S. Strite and H. Morkoc, J. Vac. Sci. Technol., B 10, 1237 (1992).

${ }^{7}$ H. Yang, L. X. Zheng, J. B. Li, X. J. Wang, D. P. Xu, Y. T. Wang, X. W. Hu, and P. D. Han, Appl. Phys. Lett. 74, 2498 (1999).

${ }^{8}$ S. H. Park and S. L. Chuang, J. Appl. Phys. 87, 353 (2000).

${ }^{9}$ P. Das and D. K. Ferry, Solid-State Electron. 19, 851 (1976).

${ }^{10}$ J. I. Pankove, Diamond, Silicon Carbide and Related Wide Bandgap Semiconductors, Edited by J. T. Glass, R. Messier, and N. Fujimori (Mater. Res. Soc. Symp. Proc., 1990), Vol. 162, p. 515.

${ }^{11}$ H. Okumura, S. Misawa, and S. Yoshida, Appl. Phys. Lett. 59, 1058 (1991).

${ }^{12}$ S. Strite, J. Ruan, Z. Li, A. Salvador, H. Chen, D. J. Smith, W. J. Choyke, and H. Morkoc, J. Vac. Sci. Technol., B 9, 1924 (1991); O. Brandt, H.
Yang, B. Jenichen, Y. Suzuki, L. Daweritz, and K. H. Ploog, Phys. Rev. B 52, R2253 (1995).

${ }^{13}$ R. X. Wang, S. J. Xu, S. Fung, C. D. Beling, K. Wang, S. Li, Z. F. Wei, T. J. Zhou, J. D. Zhang, Y. Huang, and M. Gong, Appl. Phys. Lett. 87, 031906 (2005).

${ }^{14}$ C. C. Zheng, J. Q. Ning, Z. P. Wu, J. F. Wang, D. G. Zhao, K. Xu, J. Gao, and S. J. Xu, RSC Adv. 4, 55430-55434 (2014).

${ }^{15}$ S. J. Xu, L. X. Zheng, S. H. Cheung, M. H. Xie, S. Y. Tong, and H. Yang, Appl. Phys. Lett. 81, 4389 (2002); S. J. Xu, C. T. Or, Q. Li, L. X. Zheng, M. H. Xie, S. Y. Tong, and H. Yang, Phys. Status Solidi A 188, 681 (2001).

${ }^{16}$ T. Prokofyeva, T. Sauncy, M. Seon, M. Holtz, Y. Qiu, S. Nikishin, and H. Temkin, Appl. Phys. Lett. 73, 1409 (1998).

${ }^{17}$ J. Zi, X. Wan, G. H. Wei, K. M. Zhang, and X. D. Xie, J. Phys.: Condens. Matter 8, 6323 (1996)

${ }^{18}$ M. Cardona and G. Guntherodt, in Light Scattering in Solids II, edited by M. Cardona and G. Guntherodt (Springer, Berlin, 1982), p. 46.

${ }^{19}$ A. Trampert, O. Brandt, H. Yang, and K. H. Ploog, Appl. Phys. Lett. 70, 583 (1997).

${ }^{20}$ J. L. T. Waugh and G. Dolling, Phys. Rev. 132, 2410 (1963).

${ }^{21}$ D. G. Zhao, S. J. Xu, M. H. Xie, S. Y. Tong, and H. Yang, Appl. Phys. Lett. 83, 677 (2003).

${ }^{22}$ S. J. Xu, H. Wang, Q. Li, M. H. Xie, X. C. Wang, W. J. Fan, and S. L. Feng, Appl. Phys. Lett. 77, 2130 (2000).

${ }^{23}$ J. Q. Ning, S. J. Xu, D. P. Yu, Y. Y. Shan, and S. T. Lee, Appl. Phys. Lett. 91, 103117 (2007)

${ }^{24}$ T. R. Welberry, Rep. Prog. Phys. 48, 1543 (1985). 